\title{
QUATTRO ANNI DI ISOLAMENTI DI STENOTROPHOMONAS MALTOPHILIA PRESSO IL POLICLINICO SAN DONATO.
}

\author{
Baccalini R., Tejada M., Gaia P., Ngounou A., Costa E. \\ Servizio di Medicina di Laboratorio, Policlinico San Donato, \\ San Donato Milanese.
}

Introduzione: nell'arco di quattro anni valutiamo l'andamento delle infezioni da Stenotrophomonas maltophilia per i pazienti ricoverati all'interno della struttura ospedaliera.

Materiali e metodi: dal 01 gennaio 2001 al 31 dicembre 2004 sono stati considerati tutti i casi di isolamento da materiali clinici di Stenotrophomonas maltophilia. Si è considerato un solo ceppo per paziente e per materiale clinico Tutti i ceppi sono stati identificati con le gallerie ID32E del sistema ATB (bioMérieux).

Risultati: le infezioni da Stenotrophomonas maltophilia si sono mantenute costanti nei quattro anni: 27 casi nel 2001, 29 nel 2004, con un picco di isolati nel 2002 (35). Il reparto maggiormente coinvolto risulta la Terapia Intensiva Post Operatoria (TIPO). In esso nel 2003 studiamo un calo di isolamenti con $51.7 \%$ di casi sul totale delle infezioni riscontrate nell'intera struttura ed ancora una lieve diminuzione nel 2004 (68.9\%), contro una incidenza del 77\% nel 2001 e del $74.3 \%$ nel 2002. Fra i materiali clinici di isolamento il broncoaspirato rappresenta la fonte principale seguito da espettorato, tamponi da ferita ed emocolture. Sette ceppi sono stati isolati in TIPO nel 2004 da soluzioni di lavaggio. Nell'arco dei quattro anni la proporzione delle emocolture positive è fortemente diminuita: se nel 2002 la sepsi da Stenotrophomonas maltophilia rappresentava il 20\% dei casi, nel 2003 questa percentuale risulta ridotta al $3.5 \%$ e nel 2004 non si studiano casi. Gli isolamenti da broncoaspirato risultano invece costanti nei quattro anni con una percentuale pari al $55 \%$.

Conclusioni: è ipotizzabile che l'assenza di emocolture positive nel 2004, sia il risultato di interventi mirati messi in atto 
dal CIO, tra cui: la revisione dei processi di disinfezione e sterilizzazione, la formazione specifica del personale infermieristico sul problema delle infezioni e l'attuazione di misure correttive a livello ambientale. 